\title{
METHOD OF TREATMENT OF PERIIMPLANTITIS
}

\author{
Tihomir Georgiev \\ Department of Oral and Maxillofacial Surgery \\ Medical University of Varna
}

\section{SUMMARY}

The article deals with the problem of treatment of periimplantitis which is one of the most severe complications in the implant practice of a dental doctor. The author suggests a new method of treatment of periimplantitis which is substantiated by his clinical research and observations. The clinical comparative study demonstrates the reliability of this method as compared to the conventional methods.

Key words: periimplantitis, dental lasers, general osteotropic treatment

\section{INTRODUCTION}

When making dental prostheses for patients with partial or full adentia, a serious problem remains the reliability of fixation of prostheses, attainment of uniformity in the distribution of chewing pressure. In spite of the numerous various structures on dental implants, the most severe complication related to the placement of prostheses is the occurrence of periimplantitis - an inflammatory destruction of the peri-implant tissues which is directly related to inadequate distribution of the chewing pressure on the tissues surrounding the implant, thus leading to loosening of the artificial supports, infection of the surrounding tissues and consequently - inflammatory processes. The occurrence of these phenomena reduces the survival of implants within early terms, leads to disintegration between the implant-bone connection and eventually, to atrophy of the bone and the soft tissue around the implant.

There are known methods of treatment of the inflammatory - destructive processes in the peri-implant tissues, directed towards an effect on the hard and soft tissues of the periodontium, including conservative and surgical intervention.

The use of these methods of treatment, when there is an infected wound, reduces the osteoplastic function of the bone tissue of the alveolar process of the jaw. This entails inability to completely crop and remove the inflammatory destruction of the peri-implant tissues which leads to recurrences after a certain period of time.

A popular method of treatment of the inflammatory destruction of periodontal tissues is the one suggested by Buser D., Dahlin C., Schenk R.K. - Guided Bone Regeneration (GBR) in Implant Dentistry, 1994).

The method involves removal of the granular tissue and the tartar from the periodontal pockets. After that over the cleaned region it is placed a bioinert polymer membrane which serves as a barrier against the in-growth of epithelial cords into the bony defect. According to the author's opinion, GBR eliminates the defect after 6 months.

However, the use only of a barrier membrane often leads to re-infection of the cancellous bone affecting the residual cytostatistic organic material and damage of the implant surface and the bone. In addition to that, the local application of different preparations such as antibiotics, antiseptics etc. leads only to a short-lasting improvement of the soft-tissue structures.

The objective of this paper is to improve the therapeutic effect of the treatment of periimplantitis.

AIMS:

- Regeneration of bone structures;

- Complete elimination of inflammatory processes in the peri-implant tissues;

- Reduction in the duration of treatment;

- Creation of aseptic conditions around the implant;

- Securing the reliability of the implanted artificial supports.

\section{MATERIALS AND METHODS:}

In ambulatory surgical conditions it was carried out treatment of 16 patients who have been established the diagnosis "periimplantitis". They were divided in two groups (equal in number), depending on the type of treatment. The treatment of the $1^{\text {st }}$ group was carried out in accordance with the GBR method suggested by Buser and co-authors, while in the second group the surgical treatment was implemented using laser device Waterlase MD (Er,Cr:YSGG laser) produced by Biolase Inc., USA and the patients were prescribed osteotropic medicines per os in order to assist the regeneration of the bony defect.

The author has already proved in his doctorate thesis that generalized periodontitis is very often accompanied by a disorder in the mineral density of the bone tissue of patients (osteopenia, osteoporosis). Both experimentally and clinically, the author substantiates the treatment of these periodontites by prescribing general osteotropic treatment the purpose of which is to ensure potential for the local surgical treatment. This experience and the fact that in view 
of its etiology periimplantitis is similar to periodontitis, give us grounds to apply the same method of treatment.

What is more, in Western Europe laser treatment has been discussed for a long time now in all areas of dental medicine, especially in oral surgery and periodontology. The power of dental lasers in these domains lies mostly in their ability to disinfect and decontaminate the surgical wound which on its turn is in the basis of successful treatment of periimplantitis.

During operation of the patients of $2^{\text {nd }}$ group it was used dental laser Waterlase MD (Er,Cr:YSGG laser) which is a representative of the last generation of hard lasers. The incision of tissues, cleaning of granulates, surgical treatment of the wound, as well as the sanitation of the wound was wholly carried out by means of the dental laser, changing only the various types and settings for these procedures ( laser settings are :for incision - 1,75 W, 11\% Air, 4\% Water, for treatment of the implant surface - Defocused mode, 4 W, $60 \%$ Air, 20-30\% Water, for sterilization - $5 \mathrm{~W}, 11 \%$ Air, no Water, ) i.e. the surgical wound is not in touch with other instruments, the implant surface is also decontaminated and cleaned by means of the laser.

To the patients of the 2 groups after sanitation of the surgical wounds there were placed bone material and bioinert membrane, the wounds were sutured and the stitches were removed after the $7-10^{\text {th }}$ day. When there were strongly displayed inflammatory reactions and pus, we prescribed antibiotics.
To the patients of the $2^{\text {nd }}$ group, in addition to the local surgical treatment we also applied general osteotropic treatment - we prescribed Piascledine (1 caps. per day for 1 month + Coxamine D $1000 \mathrm{mg}$ ( 1 capsule per day).

\section{RESULTS:}

With the patients of group 1 we had $50 \%$ of successful treatment of periimplantitis (in four of them infection persisted and as a result of that the implants had to be substituted for others, after the process died out).

With the patients of group 2, the successful treatment of periimplantitis was $90 \%$, during the post-surgical period the implants stood stable, there were not noticed any symptoms of inflammation, the healing process was smooth. In 1 patient, however, the inflammatory process recurred after 2 weeks.

\section{DISCUSSION OF THE RESULTS:}

The results obtained show that it is appropriate to treat periimplantitis using an Er,Cr:YSGG laser, as well as to prescribe general osteotropic medicines that enhance bone regeneration in the region of inflammatory destruction of the bone around the implant. This combination of surgical and general therapeutic treatment aims at improvement of the quality of regenerated bone structures and continuous preservation of the artificial supports in the oral cavity.

The above method enabled the author to reduce the duration of treatment and to ensure reliability of the results.

\section{REFERENCES:}

1. Arhipov V.D. - Optimization of the local treatment of the fractures of the low jaw and development of the metods for precautions of pyoinflammatory complications. The author's abstract from dissertation.1988, p.33

2. Banchenko G.V., Perova M.D. New in stomatology -1997. - Nr.6 - p.3744

3. Vuraki K.A., Vasiliev A.V.,
Nesmyanov A.A. - New in stomatology 1993. -Nr.3 - p.19-23.

4. Zubov J.I. - Pressing questions of stomatologic implantation - Minsk, 1996. - p.61-68

5. Paraskevas P.S. - Pressing questions of stomatologic implantation - Minsk, 1996. - p.35-40

6. Porteder H., Henning G. Stomatology - 1997, Nr.3 - p.26-28
7. Giovanni Serino, Christer Strom Peri-implantitis in partially edentulous patients : association with inadequate plaque control - Journal of Clinical Oral Implant Research - 2009, issue 2, 169 174

8. Klinge, B., Hultin, M., Berglundh, T. Periimplantitis. Dental clinicals of north America - 2005 49: 669 - 676

Address for correspondence:

Tihomir Georgiev

Str. Hristo Popovich Nr.18

Varna 9000 Bulgaria

e-mail: tgeorgievphd@yahoo.com 